\title{
The shifting sands of coronary practice: from cardiologist's amusement to a surgeon's avocation
}

\author{
Sundeep Mishra ${ }^{1}$
}

Received: 12 April 2017 /Revised: 28 May 2017 / Accepted: 1 June 2017 /Published online: 18 July 2017

(C) Indian Association of Cardiovascular-Thoracic Surgeons 2017

\begin{abstract}
The field of coronary artery disease therapy has evolved over a period of time from medical therapy to coronary bypass surgery to interventional cardiology. However, none of the therapies have become obsolete and there is still place for optimal medical therapy, cardiac surgery as percutaneous interventions. The need of the hour is to develop an integrated multi-disciplinary approach popularly known as heart team approach at least for complex situations whether patient or lesion related.
\end{abstract}

Keyword Heart team approach $\cdot$ PTCA $\cdot$ CABG

\section{Background}

In interventional cardiology or cardiac surgery, the debate goes back to the early 80 s when cardiologists first started treating coronary artery disease $(\mathrm{CAD})$ patients with plain balloon angioplasty but under observation of cardiac surgeons who would immediately take up for (by this time) well established conventional bypass surgery. At that time, it seemed implausible that a complex coronary lesion admixed with resistant fibrous tissue and even calcium would "open up" with mere balloon dilatation and thus coronary angioplasty remained restricted to few discrete, straightforward lesions and a hobby of some cardiologists, albeit very enthusiastic ones. Major limitations of plain old balloon angioplasty (POBA) were acute vessel closure in short term and restenosis

Sundeep Mishra

sundeepmishraihj@gmail.com

1 Department of Cardiology, AIIMS, 325 Mount Kailash Tower No 2, New Delhi, Delhi 110065, India on long term. The only attractive feature of this procedure was that it was a less invasive procedure, a goal that mankind is always searching for [1]. The next 2 decades or so saw an explosion of technology of percutaneous transluminal coronary angioplasty (PTCA) with discovery of first stents and later drug eluting stents (DES) broadening indication for PTCA culminating in explosive proliferation in the number of PTCA procedures so much so that at present number of PTCAs far exceeds the number of coronary artery bypass surgery (CABG) procedures. The surgeons on their part also shifted to less invasive techniques (off-pump, port access, minimally invasive direct coronary artery bypass techniques), more complex lesions, and even out of coronary arena. However, with shrinking of pie for surgeons, some believe that pendulum has swung to the other side, and over a course of time, cardiac surgery would remain the hobby of some cardiac surgeons. Further, this has led to a lot of bad breath among these specialists, and this is no trivial spat involving mere money and ego, but the fight is about survival, specialists attempting to hold on to their turf - or claim a bit more territory - amid the shifting sands of contemporary medicine.

\section{What is reality}

Despite all advancements in PTCA and despite all acute problems associated with surgery, it does seem that surgical revascularization if properly performed is a more effective solution (although the difference between robustness of CABG and PTCA is narrowing over a period of time). Thus, for patients with less disease and simpler, less number of lesions, there may be hardly any difference in efficacy of two approaches; PTCA being far less invasive would be clearly preferred, but for complex lesions in association with diabetes mellitus (DM), CABG may still score over PTCA. The difference may lie in ability to directly work on the lesion (surgeon) 
versus indirectly by use of a device (angioplaster) as also ability to provide an alternate channel (and therefore two routes for circulation of blood) versus only one route with PTCA. On the other hand, wherever surgery carries a higher risk due to associated co-morbid conditions, PTCA will remain a reasonable alternative.

\section{Effect of SYNTAX score}

Syntax (SX) score has emerged as a reproducible angiographic tool to predict long-term clinical outcomes in patients with left main or multi-vessel disease as also in ST-segment elevation myocardial infarction (MI) undergoing primary percutaneous coronary interventions (PCI). A high SX score ( $\geq 23 \mathrm{SX}$ ) is a good predictor of adverse cardiovascular events including cardiac death, MI, and target lesion revascularization but also short-term outcomes $[2,3]$. Cardiac surgery should definitely be preferred in this group of patients unless there are significant co-morbid conditions increasing the risk of CABG.

\section{Effect of diabetes mellitus}

Patients with DM are prone to a diffuse and rapidly progressive form of atherosclerosis, which increases their likelihood of requiring revascularization. Furthermore, the disease also modifies the individual response to arterial injury so much so that there is a much higher neo-intimal proliferation after angioplasty in this group of patients. While advances in PTCA like DES reduce some of these side effects so much so that in many patients with 1- or 2-vessel disease there may be hardly any difference between the two approaches, but for multivessel $\mathrm{CAD}$, the difference may become crucial. In this subgroup, $\mathrm{CABG}$ is not only associated with fewer recurrent events or need for re-intervention but also better survival and thus studies after studies consistently demonstrated the superiority of CABG over PTCA, use of DES notwithstanding $[4,5]$. Similarly, in other situations of profoundly virulent atherosclerosis, CABG may be the best option.

\section{Left main disease}

Although there is a long history of angioplasty in the left main (LM), starting with POBA back in the late 1970s, then bare metal stents, and now DES with interventionists getting closer and closer to surgery in terms of outcomes, LM angioplasty has remained the final frontier for interventional cardiologists. The Achilles heel of the procedure is higher restenosis rates with angioplasty, which not only translates into higher repeat revascularization rates but also that this restenotic lesion is in an area which would put a very high amount of myocardium under jeopardy so much so that this lesion may not remain as benign as it would be in another location. Furthermore, there are technical challenges as well; a large vessel (larger size of stent required), a short lesion (smaller length of stent required), more use of imaging technology (the need to get a perfect result, this being an unforgiving location) and a stent with higher radial strength, but the most challenging part is bifurcation lesion which occurs in around two thirds of LM disease. The bifurcation lesion in unprotected left main (ULM) is particularly challenging because both daughter vessels (left anterior descending and left circumflex) are important and one cannot afford to sacrifice any one of them. This often entails the use of a twostent strategy with higher side-effect rates. However, the bottom line is that mortality between PCI and CABG have been same in majority of the trials, and in at least selected groups of patients, PCI may be an acceptable, perhaps even preferred alternative to CABG, and here certainly "preferences of the patient" should count $[6,7]$. Thus, at the moment, consensus is that if there is a suitable lesion in ULM (ostial or mid-shaft), it can be intervened by either procedure done carefully but for a bifurcation lesion, CABG still seems to be a better option. That being said, all cases of ULM require the collaborative decisionmaking of a general cardiologist, interventional cardiologist, and the cardiac surgeon, the so-called heart team. On positive side, the current situation does allow more flexibility in terms of individual patient decision-making and as of now, many patients with ULM disease can be managed equally by means of two strategies of revascularization if carried out by expert and experienced teams.

\section{Other complex lesions}

Chronic total occlusions (CTO) have remained another challenge for PTCA; lower success rates and higher complication rates both long and short term [8]. While single CTO may be "fair game" for any interventional cardiologist, if there are CTOs in more than one major artery it should remain in the domain of cardiac surgeon not only because of lower efficacy and higher complication rates associated with PCI but also higher cost entailed. Sole surviving artery and very low ejection fraction are other areas with higher risk of PCI unless a suitable cardiac assist device is prophylactically used (limited by higher cost and availability), here again CABG may be the most practical and safe option [9].

\section{Complications of PCI}

Intuitively, whenever complications arise out of PCI whether acutely (unmanageable acute vessel closure, perforation or hardware embolization) or chronically (malignant restenosis), surgery will remain a solution in suitable cases.

\section{So what is the solution?}

The solution is to develop criteria (ever evolving) that carefully spell out which conditions call for which procedure and 
to make certain that the general physician who first sees this patient is knowledgeable about all treatment options: surgical, interventional, medication, lifestyle modification, and others. Later on at the time of decision-making, an integrated, multidisciplinary approach the so-called heart team approach is again desirable in these patient practices with surgeons and interventional cardiologist so that patients and their relatives do not get confused as also there is no financial incentive to push one therapy or practitioner over another.

Compliance with ethical standards No research involving human participants or animal was undertaken.

No human patient in the paper so informed consent not required.

Conflict of interest The author has no conflict of interest.

\section{References}

1. Mishra S. Does modern medicine increase life-expectancy: quest for the moon rabbit? Indian Heart J. 2016;68:19-27.
2. Sianos G, Morel MA, Kappetein AP, et al. The SYNTAX score: an angiographic tool grading the complexity of coronary artery disease. EuroIntervention. 2005;1:219-27.

3. Chaudhary S. Association of syntax score with short-term outcomes among acute ST-elevation myocardial infarction patients undergoing primary PCI. Indian Heart J. 2017;69:S 20-3.

4. Aronson D, Rayfield EJ, Chesebro JH, et al. Mechanisms determining course and outcome of diabetic patients who have had acute myocardial infarction. Ann Intern Med. 1997;126:296-306.

5. Farkouh ME, Domanski M, Sleeper LA, et al. Strategies for multivessel revascularization in patients with diabetes. N Engl J. 2012;367:2375-84.

6. Stone GW, Sabik JF, Serruys PW, et al. Everolimus-eluting stents or bypass surgery for left main coronary artery disease. N Engl J Med. 2016;375:2223-35.

7. Mäkikallio T, Holm NR, Lindsay M, et al. Percutaneous coronary angioplasty versus coronary artery bypass grafting in treatment of unprotected left main stenosis (NOBLE): a prospective, randomized, open-label, non-inferiority trial. Lancet. 2016;388:2743-52.

8. Mishra S. Language of CTO interventions - focus on hardware. Indian Heart J. 2016;68:450-63.

9. Mishra S, Chu WW, Torguson R, et al. Role of prophylactic intraaortic balloon pump in high-risk patients undergoing percutaneous coronary intervention. Am J Cardiol. 2006;98:608-12. 